\title{
Risk Factors for Unplanned Admission to the Pediatric Intensive Care Unit in Pediatric Trauma Patients
}

\author{
William Schrock ${ }^{1}$, Jodi Raymond $\mathrm{MPH}^{2}$, Matthew Landman MD, $\mathrm{MPH}^{2,3}$ \\ ${ }^{1}$ Indiana University School of Medicine; ${ }^{2}$ Riley Hospital for Children, Trauma Program; ${ }^{3}$ Indiana Uni- \\ versity School of Medicine Department of Surgery, Division of Pediatric Surgery.
}

Background and Hypothesis: A number of risk factors for unplanned pediatric intensive care unit (PICU) admission and readmission have been identified. However, little is known of the risk factors associated with unplanned admission to the PICU in pediatric trauma populations. We hypothesize specific risk factors can be identified which may be associated with unplanned admission to the PICU following traumatic injury.

Methods: For this national retrospective study, we queried the 2016 National Trauma Data Bank for patients younger than 18 years experiencing a traumatic injury requiring hospital admission from the Emergency Department (ED). We excluded patients who had experienced burn injuries. Statistically significant $(p<.05)$ risk factors for unplanned PICU admission were identified in bivariate analysis and used to build a multiple logistic regression model.

Results: Patients experiencing unplanned admission to the PICU had lower ED Glasgow Coma Scale (11.83 vs. 14.31; $<<0.001$ ), higher Injury Severity Scores (ISS) (17.96 vs. 7.32; $<<0.001$ ), and were older (age 11.35 vs. $9.60 ; p<0.001$ ). Initial ED disposition to the PICU was significantly associated with unplanned admission to the PICU $(\mathrm{p}<0.001)$. Initial ED disposition to the OR was significantly associated with unplanned admission to the PICU $(\mathrm{p}=.018)$. After multiple logistic regression, ISS $(\mathrm{p}<0.001)$, initial ED disposition to the PICU ( $\mathrm{p}=0.002)$, initial ED disposition to the OR $(\mathrm{p}=0.005)$, and older age $(\mathrm{p}=0.005)$ remained statistically significant risk factors for unplanned admission to the PICU.

Conclusion: ISS and ED disposition to the PICU, OR disposition to the PICU and age are significant risk factors for unplanned admission to the PICU in pediatric trauma. These findings will assist in identifying patients at risk for unplanned admission to the PICU, thereby reducing the adverse effects of unplanned PICU admission and ultimately improving the quality of care for pediatric trauma populations. 